\title{
Research on the Integration and Verification of Foundational Software and Hardware
}

\author{
Jing Guo, Lingda Wu, Yashuai Lv, Bo Li, and Ronghuan Yu
}

\begin{abstract}
Following the high-speed development of information technology, we increasingly attach important to the information security. The development of independent self-controlled SW\&HW (software and hardware) was advanced. Thanks to the faultiness of independent self-controlled industry, the integration and verification of foundational SW\&HW must be largely strengthened. On the basis of analyzing the whole process of integration and verification of foundational SW\&HW, the technology system was advanced, and the process, the content and the flow were provided, and the environment of integration and verification was constructed.
\end{abstract}

Index Terms-Foundational SW\&HW, integration and verification, technology system, verification flow.

\section{INTRODUCTION}

Recently, the informationize system in emphasis domain and enterprise imperative use homemade SW\&HW. It can ensure the reliability of information security, and realize to hold core technology and self-controlled informationize system. The self-developed product can ensure the credibility of information security. From the view of country stratagem security to analyze, homemade SW\&HW product have higher security than overseas kindred product. Now, many countries actively develop self-controlled foundational SW\&HW platform, such as Russia had product personal computer based on homemade Ell-Bruce processor of Russia, and computer and communication system chip used in aviation and spaceflight area [1].

Under the imperious requirement of information security, self-controlled SW\&HW gets the more and more recognition. In our several purchase laws definitely stipulate to buy homemade SW\&HW [2]. Our country startup National Major Technological Projects of "HGJ". It plans gradually extend and use homemade self-controlled foundational SW\&HW during the course of "The twelfth five-year". But, the homemade SW\&HW still need to perfect on the aspect of usability, reliability compatibility and so on. The basis of zoology environment of the whole industry is still weakness. We lack a suit of testing and verification resource of quality

Manuscript received December 20, 2014; revised April 27, 2015. The work was supported in part by the "HGJ" National Major Technological Projects under Grant No. 2013ZX01045004.

The authors are with the Science and Technology on Complex Electronic System Simulation Laboratory, Beijing, CO 101416 China (e-mail: \{gquiet, freelancer_lys, libotudou, yrh1983\}@163.com,wld@nudt.edu.cn). on combination application of homemade CPU and foundation software. It lacks deep cooperation cooperative between system integrator and CPU, terminal, operation system, office software manufacturer and so on. It leads to many quality problems at the aspect of compatibility and adapter between homemade foundational SW\&HW [3]. We especially need to meet the ability of developing quality test. It can be used to all kinds of testing and verification service. Being combined with the found bug through testing and verification and cooperating with the requirement of technology solving, we could promote the whole quality of homemade CPU and foundational software.

\section{The Whole Process ANALYsis OF INTEGRATION AND VERIFICATION}

The integration and verification needs the measure of testing. The foundational mainly include CPU, operating system, middleware, database system, office software and so on. The integration testing of foundational SW\&HW is to test the function and performance of platform that are formed by foundational SW\&HW combination, and the compatibility and interoperability between foundational SW\&HW [4]. The integration testing is based on cell testing. We test to subsystem that it is assembled with all modules according to design requirement [5]. It shows that some modules could single work, but it could not assure normal work when they are lined each other. Some problems in procedure could not be reflected at local, but it could be exposed at the whole. They effect the realization of function. So we must go on the integration and verification after the SW\&HW and the information system integration. We can analyze the whole process of integration and verification of self-controlled product, as Fig. 1.

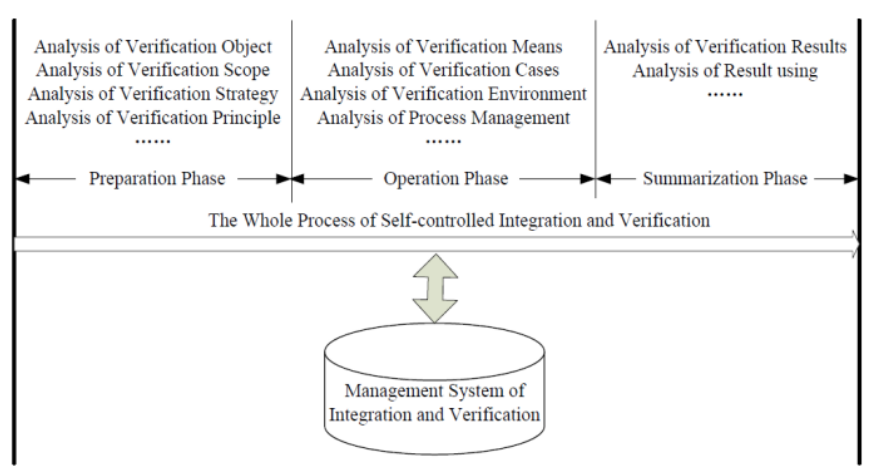

Fig. 1. The whole process of self-controlled integration and verification.

The whole process of self-controlled integration and verification mainly includes three phases. We can stipulate for the related standards to every parts. These standards will 
provide our inference and lead us to test and verify.

\section{A. Preparation Phase}

Before integrating and verifying, we should confirm the object of integration and verification, scope of integration and verification, strategy of integration and verification, principle of integration and verification, administer mechanism of negotiation. According to the related standards that we wrote, we can edit document of integration and verification aiming at the tested object. After the above elements definitude, it can lead the work to execute and promote the efficiency of verification.

\section{B. Operation Phase}

When we start to integrate and verify to product, we should ensure what environment can be constructed, how to write test case according to tested product, what means of integration and verification should choice, how to manage the test process. The writing of test case and the using of test tools are the key tache to integration and verification. In this phase, we should solve three problems of integration and verification, namely "what-how-where".

\section{Summarization Phase}

After we finish integrating and verifying to product, we should certificate the result. How can we certificate the result, it need stipulate the standard that can guarantee the scientific of verification result. In this phase, we should standardize the saving and using of the verification process document.

We could manage the process of integration and verification by using management software that we design. Its function covers with the whole process of integration and verification. We design the united and standardized flow and interface of integration and verification. It can make the test person that use the environment easily understand and quickly grip. And it can reduce the time in the process of using environment.

\section{TECHNOLOGY SYSTEM OF INTEGRATION AND VERIFICATION}

The technology system of integration and verification on foundational SW\&HW is to form a suit of negotiation and management mechanism, establish the corresponding standard, choice the advanced technology and sustainment tool. It will be modified in the operation of representative application. And it is will promote the application integration of SW\&HW. The product integration of SW\&HW include integration adaption of CPU/OS, integration adaption of foundational software, integration adaption of development and operation tool sets, integration adaption of peripheral equipment and so on. The technology system of integration and verification mainly describes the theory, standard, technology, tool, management and so on adopted in integration and verification of product. The technology system of integration and verification is as Fig. 2 [6].

We develop the integration and verification of SW\&HW. Firstly, according to software quality criterion, software test criterion, information security criterion and so on of national and military in being, and combining with test foundation, and the requirement of representative application, we establish the standard of test and evaluation. It mainly includes functionality test, reliability test, useability test, compatibility test, performance test. Secondly, we choice to study or tranfer test tool and design test cases according to test standardization system. Thirdly, according to test plan, test outline, test cases, we use the test tool of studied or transferred to execute test. Finally, according to the found problem by test and regression test, we give the test report.

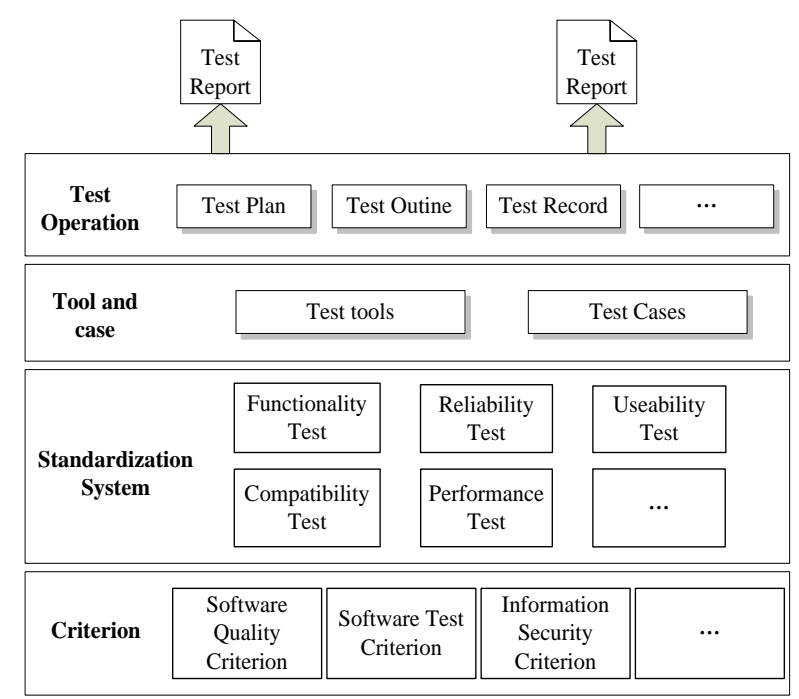

Fig. 2. The technology system of integration and verification.

Software test is a very complicated work. It can not only promote the efficiency of test work, but also enhance the stability and reliability by using test tool. It can improve the precision and veracity of test. We could finish the work that could not finish by manual test, such as load test, performance test and so on. And it can reduce the cost of work force.

The content of test tools involved in the test of functionality, security, reliability, performance, transplantable and so on. The technology development route of integration and verification tool is as Fig. 3.

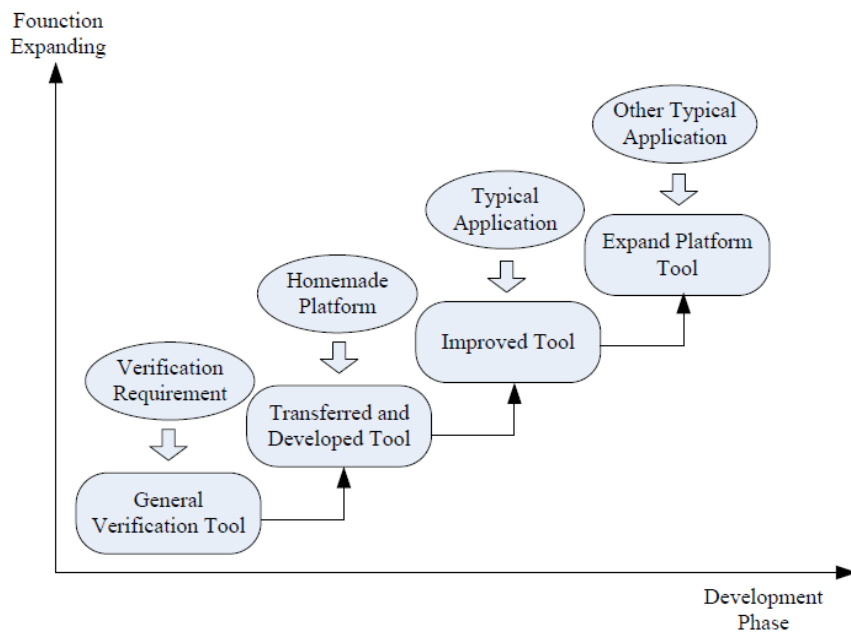

Fig. 3. The technology development route of integration and verification tool.

\section{PROCESS OF INTEGRATION AND VERIFICATION}

The integration and verification of foundational SW\&HW is involved in many technologies. The relationship between 
products is complicated. It is involved in multi-lay technologies, such the accessory construction of test environment, carding and revision of technology system. It needs various conditions to sustain. The integration testing of foundational SW\&HW is to test the function and performance of platform that are formed by foundational SW\&HW combination, and the compatibility and interoperability between foundational SW\&HW.

\section{A. Technology Framework}

Since foundational SW\&HW have many varieties, and every product has different manufacturers, and the product of different manufacturer have different varieties of editions. We use relative test software, test tool to test the function of singly foundational SW\&HW. On the basis of above work, we execute integration and verification to SW\&HW platform. Its process is as Fig. 4.

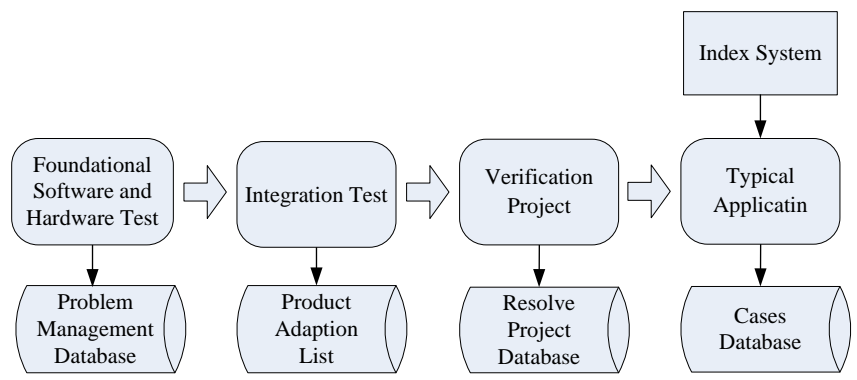

Fig. 4. The technology framework of integration and verification on SW\&HW.

Through the testing of SW\&HW, we can form problem management database. The problems which have been resolved, which unresolved and how to resolve and so on in the process of SW\&HW testing should be saved in database. In the phase of integration testing, through the adaption between many kinds of products, we can form product adaption list. Through the query of list, we can get the best adaption combination. According to the requirement of user, we test the combination of foundational SW\&HW platform, and form the adaption project and save to resolve project database. In the process, we will optimize the combination by adjusting the parameter, etc. Finally, we utilize the typical application to verify the support ability of homemade product to it. We build the index system according to the requirement of typical application and national and military standard.

The index system of typical application should consider the ability of long-time running, the ability of supporting running, the ability of easy manipulating, the function and performance of application, etc. We should define the input and output of every index.

\section{B. Test Flow}

To standardize integration test, we need establish the flow to assure the quality and efficiency of test result. The basis integration test flow [7] is as following:

\section{1) Compile test plan}

The content of test plan should be include to decide the personnel and corresponding work, test content or characteristic, advance the basis way of test, choice test means, decide the resource and technology requirement of test, make the resource plan and schedule of test.

\section{2) Compile test outline}

Combining with the characteristic of tested product, according to the general standard, criterion, we compile test outline. The test outline is the basis that test work carries out. It needs the expert to examine. The test outline is to cover with the whole function points that delegate the traits of tested product The latter test work will rigorous according as test outline.

\section{3) Deploy test environment}

Through outputting the tested product, corresponding test tool and environment software and so on from configure management database, we can deploy test environment. The building of testing environment includes the building of network establishment, the deployment of server and terminal, base software installation and test tool and so on. It can support the test of foundational SW\&HW, problem validation, edition combination optimizing, etc.

\section{4) Compile test case}

The design and execution of test case is the main body of SW\&HW test. It is the key that decide the success and defeat of SW\&HW work. The test case is to cover with the every function point in the test outline. We need design test case of integration test on the basis of quality index architecture, test criterion and appraisal criterion. The full test case should include name of test case illumination, test item, input illumination, output illumination, environment requirement, and the relation description between cases [8].

\section{5) Execute test}

Through executing test case, we can get the test result. We can check the regular or abnormity instance in the test process. If test result cannot meet termination condition of case, we can form the test problem list and then execute regression test; if test result meet condition, we terminate test. Every test point has test record. The test result should be saved to problem management database to provide query.

\section{6) Regression test}

According to the test problem list, we should execute regression test through modifying or adding the test case gather to those test case that cannot meet termination condition; we can test the modified problem by adopting previous test case, and insure no new problem derived. According to the instance of problems resolved, we go on much times test until the result is acceptable.

\section{7) Compile test report}

In the test report, the state of tested software, the difference between tested product and requirement, the found wrongs and so on should be described. Through synthesis evaluation, we finish synthesis test report. In general, the test report have template.

\section{CONSTRUCTION OF INTEGRATION AND VERIFICATION}

To meet the verification of research and achievement to integration system, a test environment should be provided. It mainly includes basis work environment, server, PC, net, etc.

Integration and verification environment need configure corresponding hardware product, such as server, desk 
terminal, network facility, safety facility and so on; corresponding foundational software, such as operating system, middleware, database system, office software and so on; testing SW\&HW, such as server for test, test tool and so on [9]. The framework of Integration and verification environment is as Fig. 5.

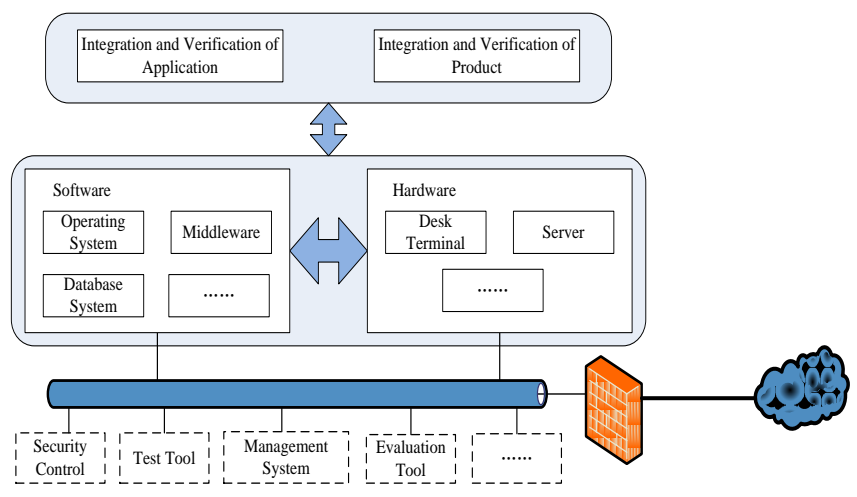

Fig. 5. The framework of integration and verification environment.

\section{A. Hardware Environment}

The environment of integration and verification includes foundational hardware and network hardware. The building of environment must fully consider testing object, referring the user' requirement for hardware choose type. Hardware need database server, application server, data backup server, mail server, MSF document server, etc. The quantity and hardware configure of server should be decided according to the application operation and requirement of testing. Since it involves in security, we need configure the corresponding security facility to guarantee the requirement of security. So we must consider the effect of performance brought by security facility and security setting.

\section{B. Software Environment}

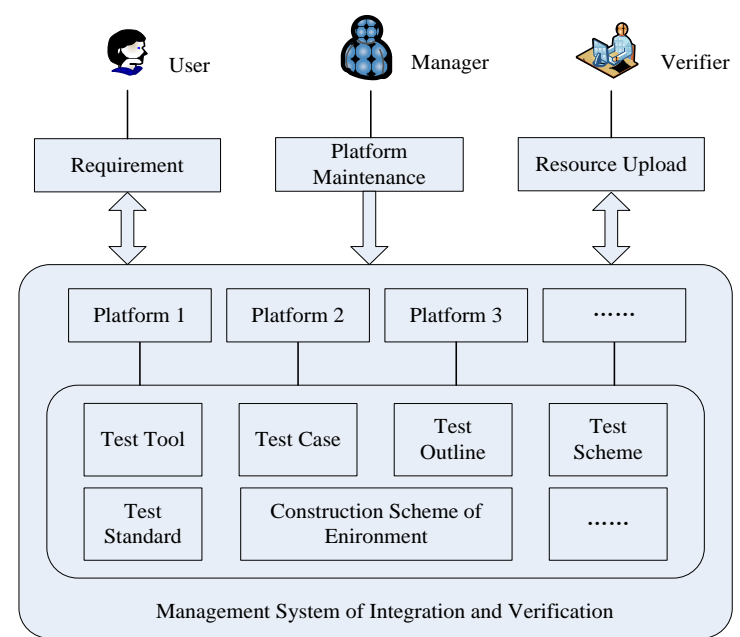

Fig. 6. The framework of management system of integration and verification.

Software environment include foundational software and testing software. The foundational software includes operating system, middleware, database system, office software, browser, mail software and so on. The choice to software type and version number should refer the user' requirement for hardware choose type. Testing software include special performance test tool, test management software and so on. We need build test casebase, test toolbase and resolve project database. The management system of integration and verification provides test requirement management, test criterion management, test operation module management, test plan management, defect management, test item, test scene, etc.

We manage the whole process by using management system of integration and verification on the homemade platform. Under the condition of environment normal operating, meeting the use purview and according with management and control of security. The user can utilize management system expediently to save, use and manage to the related results generated in the process of integration and verification. Its framework is as Fig. 6.

The management system of integration and verification adopts the pattern of homemade CPU classifying to save test tool, test case, test outline and so on. According to the requirement of integration and verification, the user looks for resource under the related CPU directory utilizing management system. The verifier save the data, self-research tool, compiled test case made in the verifying process to the related CPU directory. The manager of system provides maintenance in the light of classification.

\section{CONCLUSION}

The whole process management concept of integration and verification on foundational SW\&HW, technology system, test process, test content, test flow, environment construction being provided by the paper standardized the whole process of integration and verification. It could advance the efficiency to the integration and adaption, optimization of SW\&HW. And it could give the reference to technology integration, product integration and application integration for developing self-controlled product.

\section{ACKNOWLEDGMENT}

Jing Guo (1977-), received Ph.D. degree from National University of Defense Technology in 2008. Now she is an associate researcher in the Academy of Equipment. The direction of research is system science. Thanks to the laboratory, it provides research environment for her. And especial thank for her colleagues who gave her many helps in the process of research.

\section{REFERENCES}

[1] The ministry of industry and information technology center for international economic and technological cooperation. (2012). Russia ICT field events inventory for 2012. [Online]. Available: http://www.ccpitecc.com/article.asp?id=4435

[2] G. N. Ni, "Full spreading homemade software and hardware in electronic business," Information System Engineering, p. 12, July 2010.

[3] L. D. Wu and Y. S. Lv, "Research on the key techniques for indigenous foundational HW and SW integration," Journal of Academy of Equipment, vol. 24, pp. 1-6, 2013.

[4] J. Gao, Y. Q. Lang, and M. S. Jin, "Approach to choose integration testing combination for foundational software platform," Journal of Beijing University of Aeronautics and Astronautics, vol. 36, pp. 265-269, 2010.

[5] H. Lin, G. Ou, and W. Xiang, "Review of software testing strategy," Software Guide, vol. 7, pp. 3-4, 2008. 
[6] J. Guo and Z. W. Zhao, "Research on the integration testing of foundational software and hardware," presented at the 2014 International Conference on Vehicle \& Mechanical Engineering and Information Technology, Beijing, China, 2014.

[7] H. L. Peng, "Research on the integration testing process of embedded software and hardware," Modern Computer, vol. 9, pp. 108-111, 2010.

[8] H. L. Peng, "Research on the integration testing process of embedded software and hardware," Microcontrollers \& Embedded Systems, vol. 12, pp. 14-16, 2010.

[9] X. Z. Ren "Organization of software test and evaluation and the technique of software test," Modern Electronic Engineering, vol. 2, pp. 25-32, 2004.

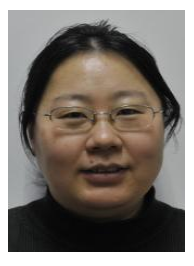

Jing Guo was born in 1977 in Jilin, China. She received her $\mathrm{PhD}$ degree in management science and engineering from National University of Defense Technology, Changsha, Hunan Province, China in 2008. Her major field of study is system programming and technology of management decision. Now she works at the Equipment Academy, China. Her main research interest is system science.

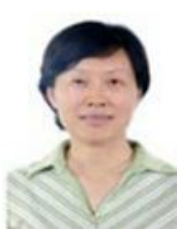

Lingda Wu was born in 1962 in Beijing, China. She received her $\mathrm{PhD}$ degree in control science and engineering from National University of Defense Technology, Changsha, Hunan Province, China in 1999. Her major field of study is virtual reality. Now she is a professor and $\mathrm{PhD}$ supervisor of the Academy of Equipment and the National University of Defense Technology. Her main research interests include multimedia information system, information processing and visualization.

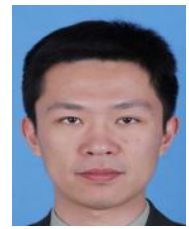

Yashuai $\mathbf{L v}$ was born in 1981 in China. He received his $\mathrm{PhD}$ degree from National University of Defense Technology in 2009. His major field of study is computer architecture. Now he works at the Academy of Equipment, China. His main research interests include processor architecture and computer graphics.

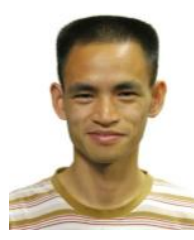

Bo Li was born in 1982 in China. He is an assistant researcher at Key Lab. of the Academy of Equipment. His research interests are mainly in system integration and network management.

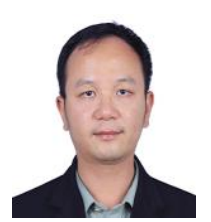

Ronghuan Yu was born in 1983 in Beijing, China. He received his $\mathrm{PhD}$ degree in control science and engineering from the National University of Defense Technology, Changsha, Hunan Province, China in 2011. His major field of study is virtual reality. $\mathrm{He}$ is an assistant of the Academy of Equipment. His main research interests include multimedia information system, information processing and visualization. 